\title{
Diagnostic Challenges of Radiation Necrosis
}

Can J Neurol Sci. 2013; 40: 763-764

Radiation necrosis (RN) may closely mimic tumor progression or recurrence (TPR) in its clinical presentation and imaging findings in brain tumor patients. In this issue, Korchi et al describe imaging appearances of five consecutive cases of RN from 73 skull-based tumors treated with proton-beam radiotherapy (RT) in Switzerland. ${ }^{1}$ An important feature of this study is the selection of patients with extra-axial tumors that tend to recur locally. As such, intra-axial lesions detected following RT more likely represent radiation injury rather than TPR — an interesting model to study imaging findings of RN. Their findings are concordant with those seen with photon RT suggesting that the process of RN may be partly independent of underlying pathology and radiation modality. This study, however, does not help us understand how to distinguish these lesions from TPR.

Although challenging, differentiating TPR from $\mathrm{RN}$ is becoming more relevant with increasing availability of newer targeted therapies and salvage RT for TPR. Conversely, patients that are accurately diagnosed with $\mathrm{RN}$ may not only be treated conservatively with steroids, but also offered hyperbaric oxygen or bevacizumab in refractory cases. A recent double-blinded randomized controlled trial showed improvement in neurological symptoms or signs in all bevacizumab-treated patients with RN. ${ }^{2}$ Although surgical resection has been the historical gold standard for diagnosis, non-invasive techniques have become increasingly preferred. Several reviews of the use of MRI, magnetic resonance spectroscopy (MRS), positron emission tomography (PET) and single photon emission CT (SPECT) in this setting have been recently published for patients with glioma. ${ }^{3-5}$

Shah et al systematically reviewed 17 studies ( 7 prospective, 10 retrospective) published between 2007 and 2012. ${ }^{3}$ Histology or clinical/radiological follow-up confirmed TPR in 282 cases (69\%) and $\mathrm{RN}$ in 100 cases (24\%). Discovery of the recurrent lesion was most often by MRI, and at a mean interval of 13.2 months following RT. Two studies $(n=104)$ of standard MRI protocols comparing lesion enhancement to the contralateral hemisphere demonstrate a cumulative sensitivity of $89 \%$ but poor specificity of $33 \%$, indicating that MRI alone appears insufficient for reliably differentiating TPR from RN.

Specialized protocols may improve performance of MRI. Dynamic susceptibility contrast enhanced (DCE) perfusion MRI measures relative cerebral blood volume (rCBV). Radiation necrosis shows low perfusion, whereas the high metabolic activity and angiogenesis of TPR results in high rCBV. Pseudoresponse from bevacizumab, an antiangiogenic agent used in the management of glioblastoma, may limit interpretability of perfusion MRI. Six studies $(n=136)$ measuring rCBV within the lesion yield cumulative sensitivity and specificity of $80 \%$ and $77 \%$, respectively. ${ }^{3}$ Vascular permeability measured as $\mathrm{K}^{\text {trans }}$ yields unimpressive results. ${ }^{3}$ Diffusion- weighted imaging (DWI) allows the microscopic diffusion of water molecules to be quantified in magnitude as the apparent diffusion coefficient (ADC), and the directionality of these water movements may be assessed by diffusion tensor imaging (DTI). Commonly, RN increases water mobility, whereas high cellular density in TPR restricts water mobility. Surrounding edema may confound DWI results, perhaps explaining the poor specificity of ADC. ${ }^{6}$

On MRS, RN shows reduced N-acetyl-aspartate (NAA) with variable changes in choline $(\mathrm{Cho})$ and creatine $(\mathrm{Cr})$, whereas high Cho is most consistent with TPR. Lactate (Lac) and lipid peaks may be present in RN. Magnetic resonance spectroscopy is limited when in close proximity to bone or air, and its spatial resolution is limited to about $1 \mathrm{~cm}$. Rock et al compared MRS with DWI to histology from image-guided biopsies in 18 patients. ${ }^{7}$ Pure tumor was reliably distinguished from pure necrosis with various metabolite ratios such as $\mathrm{Cho} / \mathrm{Cr} \geq 2.23$ (odds ratio $[\mathrm{OR}], 13.56)$ and $\mathrm{Cho} / \mathrm{NAA} \geq 0.81$ (OR, 8.75), and odds ratios increased with the inclusion of ADC for NAA/Cho $\leq$ 0.54 and $\mathrm{NAA} / \mathrm{Cr} \leq 0.67$. To distinguish pure necrosis from a mixed lesion, NAA/Cho $>1.32(\mathrm{OR}, 5.84)$ and NAA/Cr $>1.56$ (OR, 6.60) were useful. Weybright et al reported that when Cho/Cr or Cho/NAA $>1.8$ were taken to indicate TPR, 27 of 28 patients in their study were correctly classified. ${ }^{8}$ Four studies $(\mathrm{n}=66)$ of MRS yield a cumulative sensitivity and specificity of $86 \%$ and $80 \%$, respectively, with a mean Cho/NAA cut-off of 1.07. ${ }^{3}$ Magnetic resonance spectroscopy appears to be more sensitive (89-100\%) months following RT but more specific $(100 \%)$ if performed six weeks after RT. ${ }^{4}$

Positron emission tomography with $18 \mathrm{~F}$-fluorodeoxyglucose (FDG) was found to low cumulative sensitivity (70\%) and specificity $(65 \%)$ in four studies $(n=33)$ and is not recommended to reliably differentiate TPR and RN by Shah et al. ${ }^{3}$ Novel tracers have produced encouraging results. Amino acid tracers are particularly useful due to high uptake in proliferating tumors but not surrounding normal brain or RN. Five studies $(n=100)$ of 11C-methinionine (MET)-PET yield higher cumulative sensitivity (84\%) and specificity (82\%), ${ }^{3}$ and found in one study to result in a beneficial change in treatment in $36 \%$ of cases. ${ }^{9}$ However, the short half-life of 11C-MET ( 20 minutes) requires it to be produced at an on-site cyclotron, limiting its widespread clinical availability. Two PET studies with $O-(2-[18 \mathrm{~F}]$ fluoroethyl)-L-tyrosine (FET), an amino acid tracer with longer half-life ( 110 minutes), showed impressive sensitivity (100\%) and specificity (93-100\%). ${ }^{10,11}$ The nucleoside tracer 3'-deoxy3'-18F-fluorothymidine (FLT) is a more specific marker of proliferation, and demonstrated sensitivity and specificity of $100 \%$ in a small study of 25 cases $^{12}$ but offered no advantage over FDG-PET in another series. ${ }^{13}$

Single photon emission CT provides a snapshot of cerebral blood flow, with lack of metabolic activity leading to low uptake 
in RN. High cumulative specificity (98\%), while maintaining sensitivity $(88 \%)$, was seen in four studies of SPECT $(n=138){ }^{3}$ Shah et al conclude that SPECT may be the best single modality to distinguish TPR and RN due to cumulative specificity appearing higher than MRI, MRS, FDG- and MET-PET. Unfortunately, studies of FET- and FLT-PET were not included in this systematic review.

Results of combinations of MRI, FDG-PET and SPECT were reported under blinded conditions by Gómez-Río et al in a prospective study of 76 patients. ${ }^{14}$ Sensitivity was higher when MRI and SPECT were reviewed together (96\%) compared to MRI and PET together $(78 \%)$ and SPECT alone $(93 \%)$. Specificity was higher in combination imaging $(91 \%$ with MRI/SPECT and 95\% with MRI/PET) compared to SPECT alone $(86 \%)$. Sensitivity and specificity were higher in patients with high-grade glioma (tumor prevalence, $80 \%$ ) compared to low-grade glioma (tumor prevalence, 63\%) in all subgroups. Future studies of combinations of routinely-available modalities may have improved clinical applicability over exotic single modalities, if shown to provide comparable performance.

Major limitations are outlined by many authors of papers on this topic, and Korchi et al's paper in this issue is no exception. ${ }^{1}$ Studies are often retrospective, have small sample size, and involve patients only from a single center. There is lack of uniform histologic confirmation of diagnosis. Imaging protocols are not standardized for image acquisition and post-processing. ${ }^{3}$ Mixed lesions, such as rapidly-proliferating tumor with central necrosis, further complicate imaging assessments. Studies are often not adequately powered to examine statistical significance of differences in performance between imaging modalities.

These limitations remind physicians to seek all available clinical information to care for patients effectively. Risk factors such as smoking status, hypertension, diabetes mellitus, and use of concurrent chemotherapy should be reviewed. The Radiation Oncologist may be consulted to determine if the lesion lies within the high-dose regions of RT. Increasing use of modern techniques such as intensity-modulated RT and volumetricmodulated arc therapy may lead to an unexpected distribution of high-dose islands of radiation. Co-registration of the current diagnostic scan showing the lesion with the previously treated RT plan is helpful in this regard. While MRI alone appears insufficient for reliably differentiating glioma recurrence from $\mathrm{RN}$, review of the interval after RT before appearance of the lesion and lesion kinetics (regression with time for RN) may add value. Supplementary imaging may provide valuable information, noting that remarkable specificity is seen with FETand FLT-PET, SPECT, and MRS, but robust comparative studies between these modalities are not available.

Samir Patel, Vivek Mehta Divisions of Radiation Oncology and Neurosurgery University of Alberta, Edmonton, Alberta, Canada Email: samir.patel2@albertahealthservices.ca

\section{REFERENCES}

1. Korchi AM, Garibotto V, Lovblad K-O, Haller S, Weber DC. Radiologic patterns of necrosis after proton therapy of skull base tumors. Can J Neurol Sci. 2013;40(6):800-6.

2. Levin VA, Bidaut L, Hou P, et al. Randomized double-blind placebo-controlled trial of bevacizumab therapy for radiation necrosis of the central nervous system. Int J Radiat Oncol Biol Phys. 2011;79:1487-95.

3. Shah AH, Snelling B, Bregy A, et al. Discriminating radiation necrosis from tumor progression in gliomas: a systemic review what is the best imaging modality? J Neurooncol. 2013;112: 141-52.

4. Caroline I, Rosenthal MA. Imaging modalities in high-grade gliomas: pseudoprogression, recurrence, or necrosis? J Clin Neurosci. 2011;19:633-7.

5. Rahmathulla G, Marko NF, Weil RJ. Cerebral radiation necrosis: a review of the pathobiology, diagnosis and management considerations. J Clin Neurosci. 2013;20:485-502.

6. Cha J, Kim ST, Kim HJ, et al. Analysis of the layering pattern of the apparent diffusion coefficient (ADC) for differentiation of radiation necrosis from tumour progression. Eur Radiol. 2013; 23:879-86.

7. Rock JP, Scarpace L, Hearshen D, et al. Associations among magnetic resonance spectroscopy, apparent diffusion coefficients, and image-guided histopathology with special attention to radiation necrosis. Neurosurgery. 2004;54:1111-17.

8. Weybright P, Sundgren PC, Maly P, et al. Differentiation between brain tumor recurrence and radiation injury using MR spectroscopy. AJR Am J Roentgenol. 2005;185:1471-6.

9. Yamane T, Sakamoto S, Senda M. Clinical impact of (11)Cmethionine PET on expected management of patients with brain neoplasm. Eur J Nucl Med Mol Imaging. 2010;37:685-90.

10. Rachinger W, Goetz C, Pöpperl G, et al. Positron emission tomography with O-(2-[18F]fluoroethyl)-L-tyrosine versus magnetic resonance imaging in the diagnosis of recurrent gliomas. Neurosurgery. 2005;57:505-11.

11. Pöpperl G, Götz C, Rachinger W, Gildehaus FJ, Tonn JC, Tatsch K. Value of O-(2-[18F]fluoroethyl)-L-tyrosine PET for the diagnosis of recurrent glioma. Eur J Nucl Med Mol Imaging. 2004:31:1464-70.

12. Chen W, Cloughesy T, Kamdar N, et al. Imaging proliferation in brain tumors with 18 F-FLT PET: comparison with 18 F-FDG. J Nucl Med. 2005;46:945-52.

13. Enslow MS, Zollinger LV, Morton KA, et al. Comparison of F-18 fluorodeoxyglucose and F-18 fluorothymidine positron emission tomography in differentiating radiation necrosis from recurrent glioma. Clin Nucl Med. 2012;37:854-61.

14. Gómez-Río M, Rodríguez-Fernández A, Ramos-Font C, LópezRamírez E, Llamas-Elvira JM. Diagnostic accuracy of 201Thallium-SPECT and 18F-FDG-PET in the clinical assessment of glioma recurrence. Eur J Nucl Med Mol Imaging. 2008;35:966-75 